\title{
Jean-Jacques Chalifoux
}

Professeur, Département d'anthropologie, Université Laval

(1985)

\section{"Terrains en Guyane}

\section{française et sur les hauts \\ plateaux du Nigéria”}

\author{
Un document produit en version numérique par Jean-Marie Tremblay, bénévole, \\ professeur de sociologie au Cégep de Chicoutimi \\ Courriel: jean-marie_tremblay@uqac.ca \\ Site web pédagogique : http://www.uqac.ca/jmt-sociologue/ \\ Dans le cadre de: "Les classiques des sciences sociales" \\ Une bibliothèque numérique fondée et dirigée par Jean-Marie Tremblay, \\ professeur de sociologie au Cégep de Chicoutimi \\ Site web: http://classiques.uqac.ca/
}

Une collection développée en collaboration avec la Bibliothèque

Paul-Émile-Boulet de l'Université du Québec à Chicoutimi

Site web: http://bibliotheque.uqac.ca/ 


\section{Politique d'utilisation de la bibliothèque des Classiques}

Toute reproduction et rediffusion de nos fichiers est interdite, même avec la mention de leur provenance, sans l'autorisation formelle, écrite, du fondateur des Classiques des sciences sociales, Jean-Marie Tremblay, sociologue.

Les fichiers des Classiques des sciences sociales ne peuvent sans autorisation formelle:

- être hébergés (en fichier ou page web, en totalité ou en partie) sur un serveur autre que celui des Classiques.

- servir de base de travail à un autre fichier modifié ensuite par tout autre moyen (couleur, police, mise en page, extraits, support, etc...),

Les fichiers (.html, .doc, .pdf., .rtf, .jpg, .gif) disponibles sur le site Les Classiques des sciences sociales sont la propriété des Classiques des sciences sociales, un organisme à but non lucratif composé exclusivement de bénévoles.

Ils sont disponibles pour une utilisation intellectuelle et personnelle et, en aucun cas, commerciale. Toute utilisation à des fins commerciales des fichiers sur ce site est strictement interdite et toute rediffusion est également strictement interdite.

L'accès à notre travail est libre et gratuit à tous les utilisateurs. C'est notre mission.

Jean-Marie Tremblay, sociologue

Fondateur et Président-directeur général, LES CLASSIQUES DES SCIENCES SOCIALES. 
Cette édition électronique a été réalisée par Jean-Marie Tremblay, bénévole, professeur de sociologie au Cégep de Chicoutimi à partir de :

\section{Jean-Jacques Chalifoux}

Professeur, Département d'anthropologie, Université Laval

“Terrains en Guyane française et sur les hauts plateaux du Nigéria”.

Un chapitre publié dans l'ouvrage sous la direction de Serge Genest, La passion de l'échange : terrains d'anthropologues du Québec., chapitre 6, pp. 109134. Montréal : Gaetan Morin, Éditeur, 1985, 309 pp.

[Autorisation formelle accordée par l'auteur le 17 septembre 2007 de diffuser cette œuvre dans Les Classiques des sciences sociales.]

Courriel : $\quad$ Jean-Jacques.Chalifoux@ant.ulaval.ca ou jean.jacques.chalifoux@videotron.ca

Polices de caractères utilisée :

Pour le texte: Times New Roman, 14 points.

Pour les citations : Times New Roman, 12 points.

Pour les notes de bas de page : Times New Roman, 12 points.

Édition électronique réalisée avec le traitement de textes Microsoft Word 2004 pour Macintosh.

Mise en page sur papier format : LETTRE (US letter), 8.5'” x 11’')

Édition numérique réalisée le 29 février 2008 à Chicoutimi, Ville de Saguenay, province de Québec, Canada. 
Jean-Jacques Chalifoux

Professeur, Département d’anthropologie, Université Laval

\section{"Terrains en Guyane française et sur les hauts plateaux du Nigéria”}

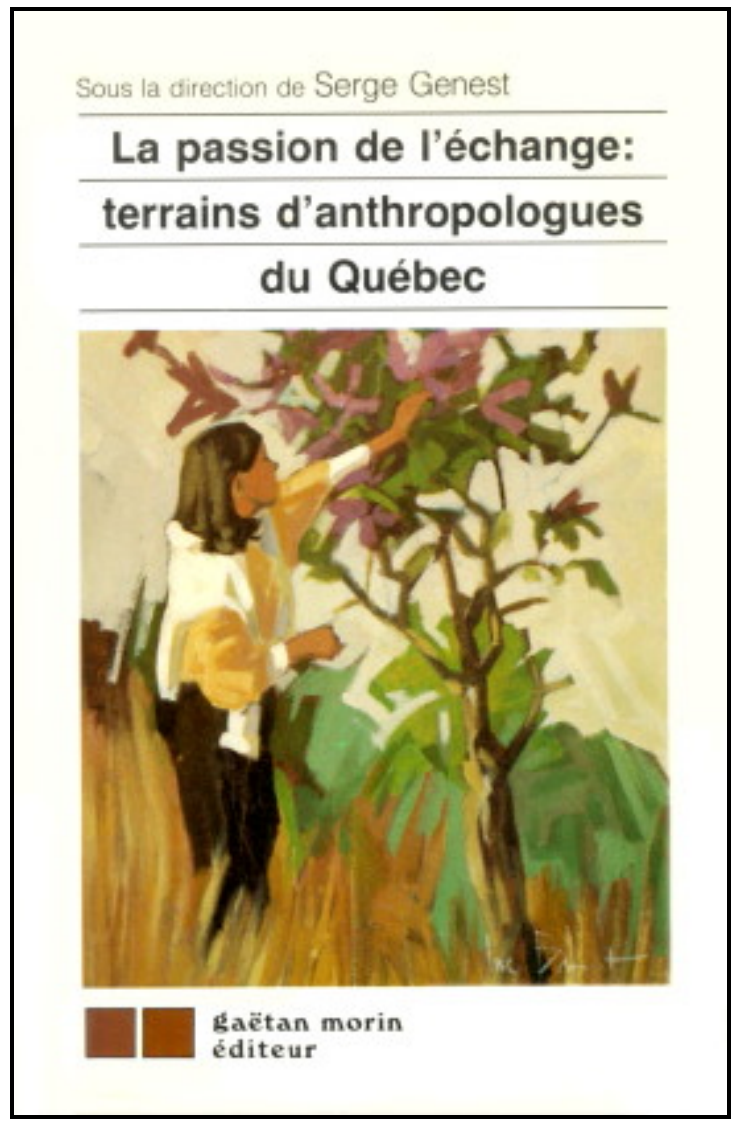

Un chapitre publié dans l'ouvrage sous la direction de Serge Genest, La passion de l’échange : terrains d'anthropologues du Québec., chapitre 6, pp. 109-134. Montréal : Gaetan Morin, Éditeur, 1985, 309 pp. 


\section{Table des matières}

$\underline{\text { Introduction }}$

«Prêt ou pas prêt, j'y vais... »

Nourritures terrestres

L'ethnographie

$\underline{\text { Conclusion }}$

Photo 1. Ces femmes qui ont plusieurs maris.

Photo 2. La Guyane française

Photo 3. Localisation géographique des Abisi au Nigéria 


\section{Jean-Jacques Chalifoux \\ Professeur, Département d’anthropologie, Université Laval}

“Terrains en Guyane française et sur les hauts plateaux du Nigéria”.

Un chapitre publié dans l'ouvrage sous la direction de Serge Genest, La passion de l'échange : terrains d'anthropologues du Québec., chapitre 6, pp. 109134. Montréal : Gaetan Morin, Éditeur, 1985, 309 pp.

\section{Introduction}

$\underline{\text { Retour à la table des matières }}$

L'objet de ce texte est de faire état du vécu de deux expériences de recherche sur le terrain, l'une en Amérique du Sud et l'autre en Afrique. Il s'agit d'un exercice à caractère autobiographique dont l'analyse déborde l'énonciation de faits. Quand un informateur se remémore son passé, il raconte son vécu tel qu'il le perçoit et le conçoit au moment actuel et le réaménage de façon à lui donner un sens aujourd'hui. L'exposé qui suit n'échappe pas à ce mélange de réalisme et d'interprétation.

Dans un premier temps, les différences dans la préparation des terrains seront présentées de manière à montrer comment la flexibilité est souvent une condition de réussite. Ensuite, après avoir exposé certains éléments relatifs à la vie quotidienne de chaque endroit, seront expliquées les conditions mêmes des enquêtes.

Le principal contraste qui se dégage de ces deux terrains peut se résumer ainsi : le premier est dominé par une expérience mystique personnelle créée par une relation directe entre deux personnes, alors que le second est caractérisé par une démarche plus positiviste et impersonnelle. 


\section{Ces femmes qui ont plusieurs maris.}

$\underline{\text { Retour à la table des matières }}$

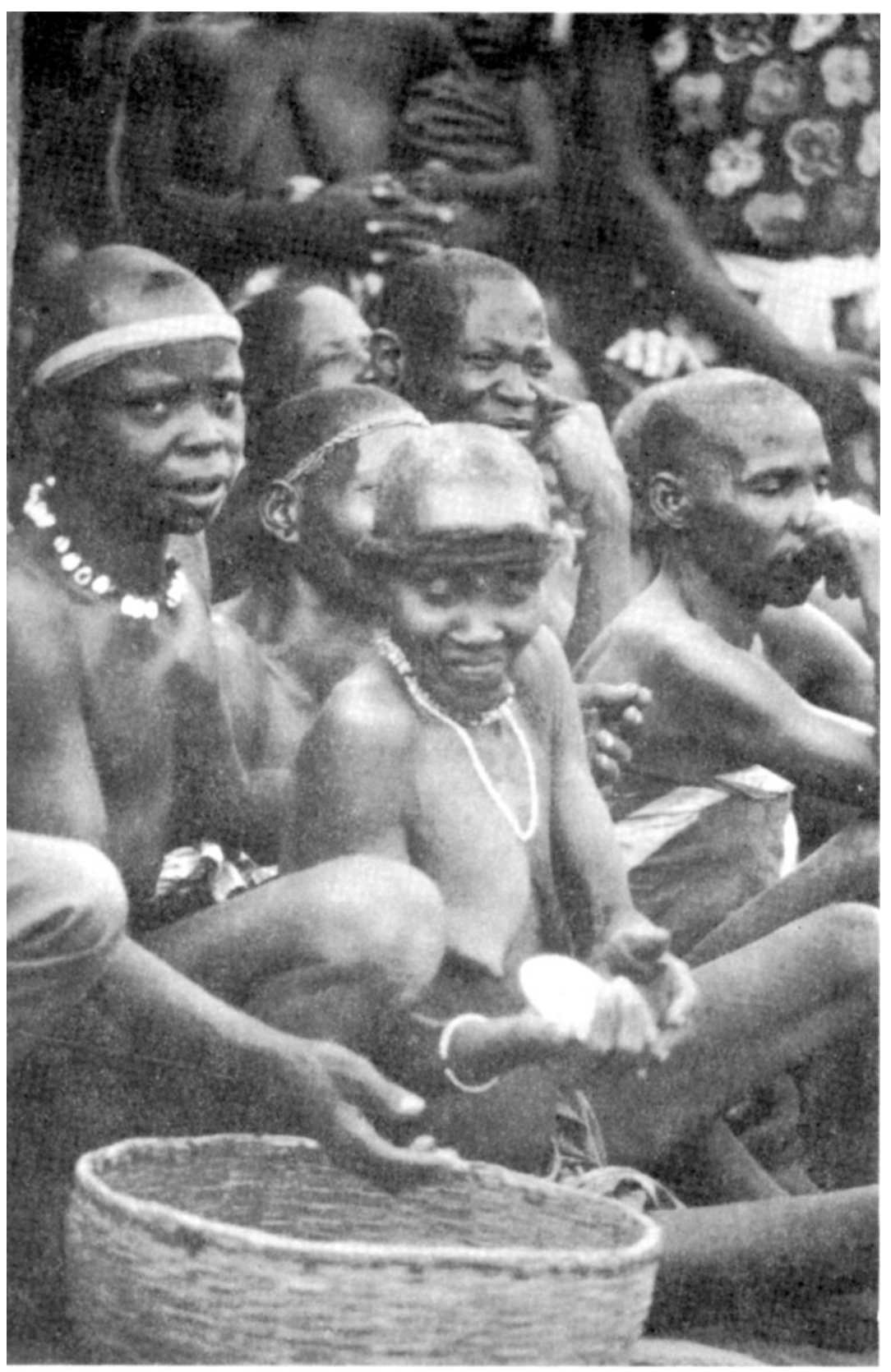

Ces femmes qui ont plusieurs maris.

$\underline{\text { Illustration dans Les Classiques des sciences sociales. }}$ 
On peut reconnaître plusieurs genres de relations ethnologiques. L'une concerne les informateurs clés qui sont des personnes avec lesquelles l'ethnographe poursuit une relation privilégiée à long terme et dont les connaissances permettent d'établir un dialogue critique et relativiste. Dans les meilleurs cas, l'informateur ou l'informatrice possède une expérience pédagogique au sein de sa propre culture ; sa position sociale et son rang comportent des devoirs dans la transmission du savoir de sa société. Cette personne peut être un-e meneur ou meneuse ou un-e marginal-e, mais dans les deux cas, elle maîtrise un discours global sur sa société et accepte d'enseigner ce savoir à l'ethnologue. La première expérience dont il s'agit concerne une catégorie particulière d'informateur clé appelée gourou dans plusieurs civilisations orientales. Dans ce cas, il s'agit d'un gourou d'origine javanaise ayant émigré en Guyane française (Amérique du Sud), avec qui j'ai vécu quatre mois en 1968 et que j'ai visité par la suite, en 1981 et 1982. L'autre recherche s'est déroulée au Nigéria au cours de l'année 1973. Là aussi, il y eut quelques informateurs clés, mais les informations furent davantage diversifiées et résultèrent surtout d'enquêtes systématiques : questionnaires, recensements, observations, compilations.

\section{« PRÊT OU PAS PRÊT, J'Y VAIS ... »}

\section{Chez les Indonésiens de la Guyane française}

$\underline{\text { Retour à la table des matières }}$

Au cours des années 60, au Département d'anthropologie de l'Université de Montréal, sous la direction de Jean Benoist, émergeait un centre d'études consacré aux civilisations des Caraïbes.

L'un des projets de recherche concernait la génétique des populations humaines et les Îles Antillaises peuplées d'immigrants d'Amérique, d'Asie, d'Afrique et d'Europe constituaient un laboratoire idéal. Ce projet m'intéressait particulièrement car, après avoir terminé un $\mathrm{B}$. Sc. en biologie et enseigné au cégep, je cherchais à me renouveler intellectuellement. Comme la génétique m'avait particulièrement inté- 
ressé au cours de ma formation en biologie, je découvris que le Département d'anthropologie de l'Université de Montréal était l'endroit pour poursuivre cette réflexion. Des médecins et des biologistes ayant une formation complémentaire en ethnologie travaillaient conjointement afin de rapprocher ces disciplines.

\section{La Guyane française}

$\underline{\text { Retour à la table des matières }}$

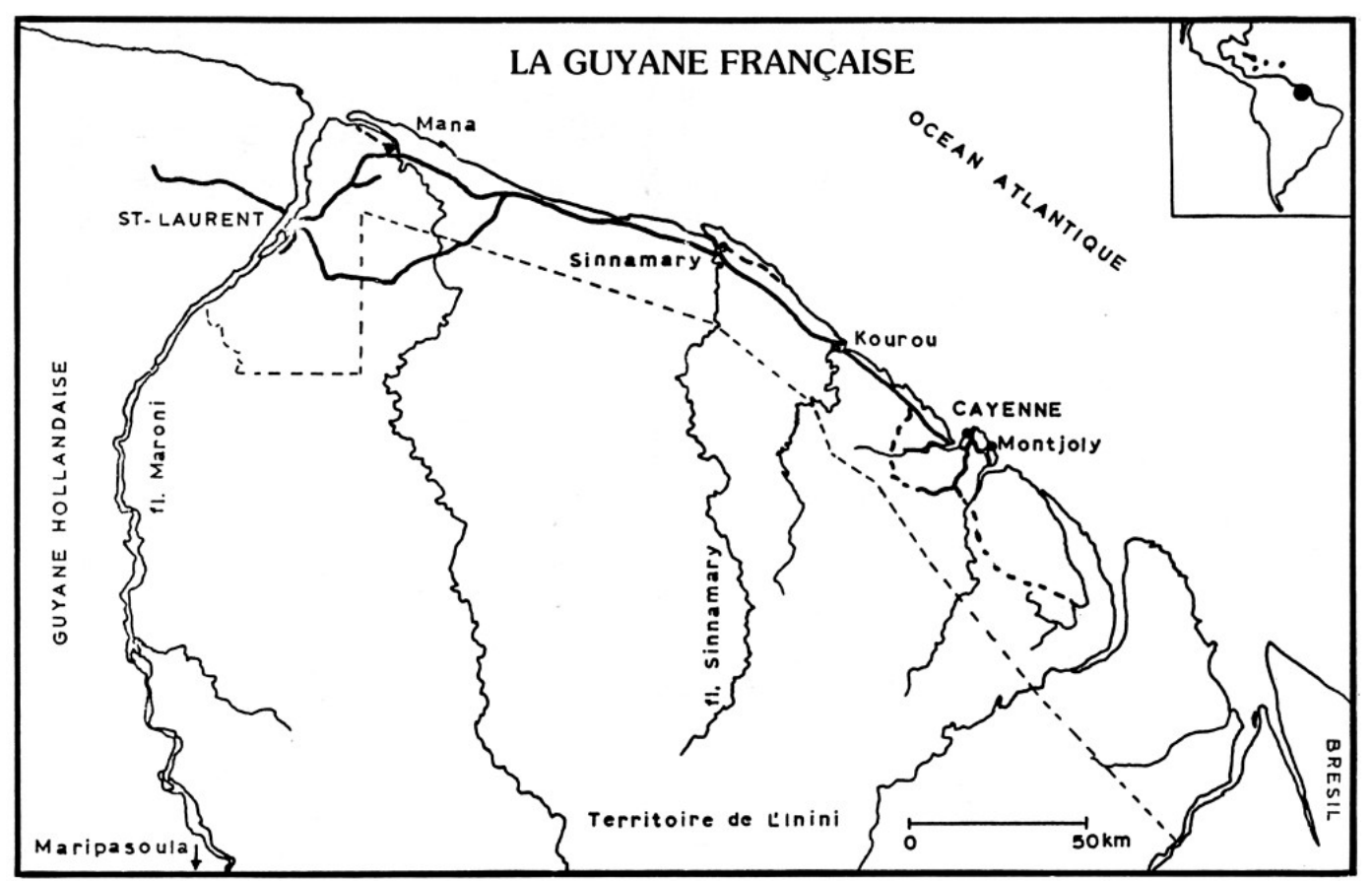

$\underline{\text { Illustration dans Les Classiques des sciences sociales. }}$

Au printemps de 1968, ma scolarité de maîtrise en anthropologie terminée (après deux années de recyclage), je m'apprêtais à partir pour la Martinique détenteur d'une bourse d'études de l'« Institute for the Study of Man » de New York. Ce projet était encadré par un médecinanthropologue et portait sur l'étude du métissage. Deux semaines avant le départ, $M$. Demetz était trouvé mort dans sa petite chambre de l'ancienne rue Maplewood. Le projet ne pouvait plus être réalisé par un étudiant seul. Toutefois cet incident de parcours me donna la possibilité de voler de mes propres ailes. Le directeur du Centre de 
recherches Caraïbes accepta d'emblée que je présente un projet de recherche et, comme je rêvais de l'Amazonie depuis longtemps, je proposai de réaliser une anthropométrie des Indiens Galibi de la côte de la Guyane française.

Si je me rappelle bien, ma bourse était de 900\$. En déduisant $500 \$$ pour le transport, il ne me restait que $25 \$$ par semaine pour vivre et travailler, un strict minimum même à cette époque. Je débarquai donc à Cayenne, seul et sans aucune relation préétablie. Pour un dollar par jour, je louai un lit dans un dortoir d'hôpital et préparai le voyage chez les Galibi, installés à quelque $300 \mathrm{~km}$ plus loin, près de Mana. Tout d'abord, je me présentai à la préfecture et quelques minutes plus tard, j'avais une autorisation de séjour. Ensuite, après avoir noué une amitié avec un patient de l'hôpital d'ethnie galibi, nous avions décidé de faire la route ensemble grâce à un automobiliste qui se rendait à mi-chemin entre Mana et Sinnamary.

Mon compagnon m'apprit alors que beaucoup de gens avaient quitté temporairement leur village pour travailler à la construction d'une base spatiale européenne. Comme mon projet exigeait un échantillonnage scientifique d'hommes, je compris que cette migration pouvait menacer la méthodologie du projet. Arrivés à Sinnamary, nous allâmes manger, à peu de frais, dans une famille qui, les samedi et dimanche, faisait office de restauration à domicile.

La petite maison de bois avait été construite par son propriétaire. Elle était entourée d'un magnifique jardin tropical et située près d'un pont de bois donnant accès à un îlot où s'étaient installées quelques centaines de personnes, dans une vingtaine de maisons. L'atmosphère de ce village était extraordinaire : la végétation, l'esthétique des plantations, l'architecture particulière et la population composaient une petite communauté comme celles dont rêvaient les ethnologues des années 60. Il s'agissait de paysans nés à Java et amenés par les colonisateurs hollandais au Surinam (l'ancienne Guyane hollandaise), en Amérique du Sud. Plus tard, ces immigrants auraient décidé de s'installer chez leur voisine la Guyane française, où leur compétence en riziculture convenait parfaitement aux plans de développement économique de la région. 
En conversant avec le restaurateur qui était aussi le Lurah, chef ou maire, du village et connu en Guyane comme un homme affable et hospitalier, j'appris quelques particularités sur la communauté. Papa chef, ainsi qu'on l'appelle, et sa femme comprirent rapidement ma motivation et acceptèrent que je réside chez eux moyennant une pension proportionnelle à mes moyens. Par pur opportunisme, sans problématique spécifique, mais avec la latitude offerte par le directeur du Centre de recherches Caraïbes, j'amorçai une réflexion ethnologique qui allait me conduire quelques années plus tard en Inde d'où mon intérêt marqué pour les civilisations orientales.

Au cours de ce terrain, je me limitai exclusivement à ce village. Au milieu de mon séjour, je reçus la visite de mon directeur de thèse qui fut tout à fait satisfait, mais surpris, de la tournure des événements qui venait confirmer sa théorie de l'« eau bouillante » (sic). La technique est relativement simple. Elle consiste à placer les personnes dans un contexte d'enquête donné et elles doivent fournir le maximum d'ellesmêmes. Si elles réussissent à s'en sortir, c'est le succès ; sinon, c'est l'échec. En fait, cette surprise, je me la fis d'abord à moi-même, car cette expérience fut le centre de mon intérêt pour la génétique et l'anthropologie socioculturelle. En effet, pendant que je collectais les données socio-démographiques et que j'allais d'une famille à l'autre pour réaliser mes 76 mesures anthropométriques par personne, je recueillais aussi toutes les observations possibles, je discutais de tout ce que je ne comprenais pas et surtout je développais une réflexion mystique avec le chef gourou. Tranquillement, mais sûrement, l'ethnologie devenait la démarche qui M'importait le plus. Elle contrastait avec la rigueur des exigences des méthodes de l'anthropométrie qui vous font pénétrer dans l'intimité des gens à la manière d'un médecin, mais sans obtenir la contrepartie immédiate du traitement !

Cette improvisation est-elle si dramatique que cela ? Est-il toujours vraiment nécessaire d'aborder une recherche selon les règles de la science sociologique qui exige une prévoyance hypothético-déductive à toute épreuve et qui, souvent, oriente plusieurs chercheurs dans les domaines déjà établis de la recherche des autres ? En fait, la formation anthropologique québécoise fournit des cadres intellectuels généraux amplement suffisants pour qu'un jeune ethnographe puisse découvrir 
des problèmes inédits et puisse les relier aux contextes historique, politique et culturel de la région étudiée. Le reste est une question de labeur et de personnalité. De plus, à long terme, le recul hors terrain permet de confronter les idées développées sur place à celles des autres chercheurs qui ont réfléchi aux mêmes questions. À cet égard, le retour éventuel sur le terrain constitue une démarche idéale, car non seulement on apprend des autres ethnologues, mais le temps contribue à l'approfondissement des relations interpersonnelles.

\section{Localisation géographique des Abisi au Nigéria}

$\underline{\text { Retour à la table des matières }}$

\section{LOCALISATION GÉOGRAPHIQUE DES ABISI AU NIGÉRIA}
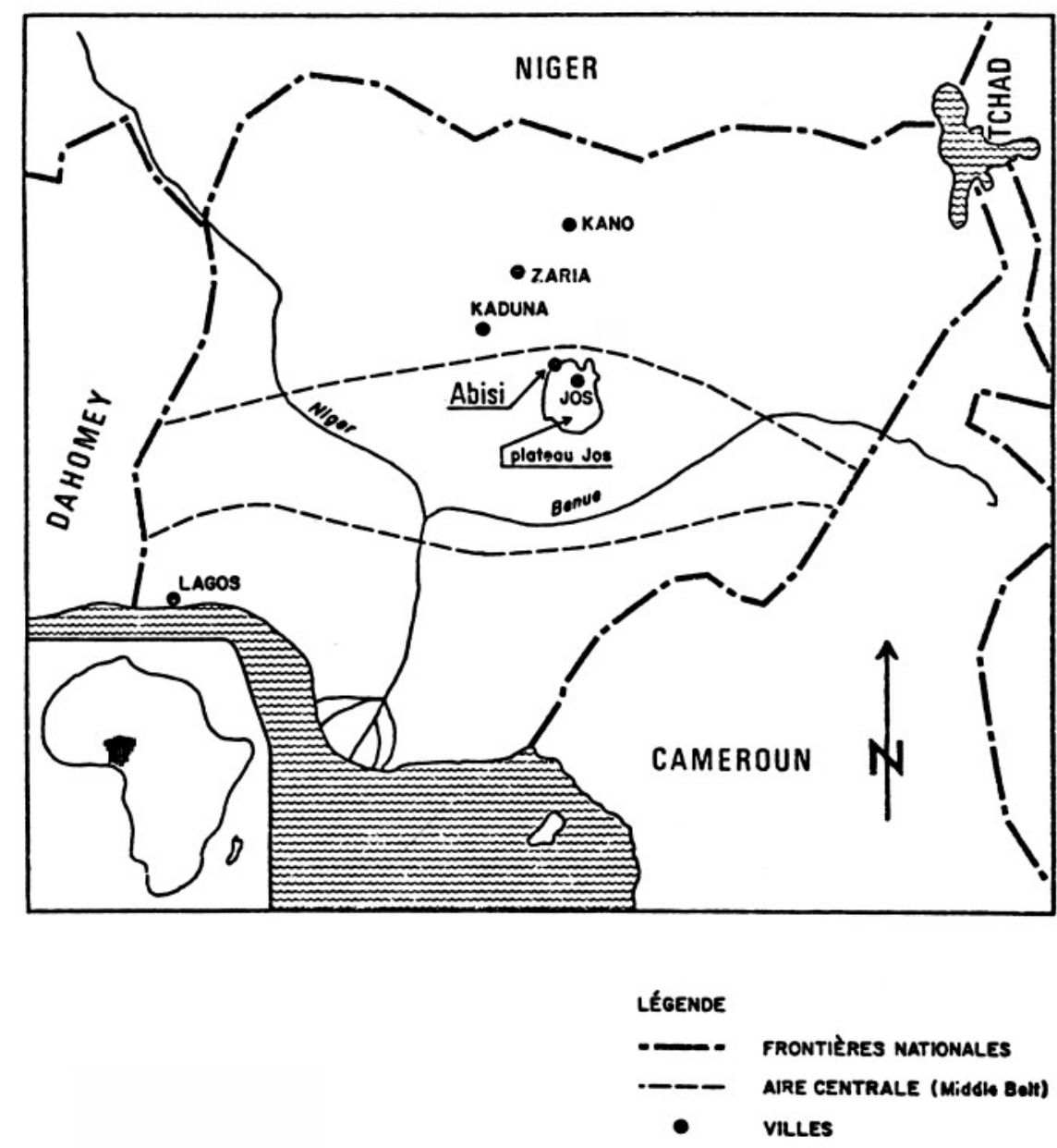

$\underline{\text { Illustration dans Les Classiques des sciences sociales. }}$ 
Pour illustrer la remarque précédente, mes séjours en 1980 et 1981 ont renforcé mes liens d'amitié de telle façon que l'amie qui m'accompagnait la dernière fois fut en quelque sorte considérée comme une " soeur » ou une " bru » et de ce fait, encouragée à participer aux travaux de la maison. Notre « mère » lui relatait d'ailleurs avec satisfaction comment lors de mon premier séjour, « Jean-Jacques était maigre et pauvre comme nous ", mais que douze ans plus tard j'étais devenu " gras et riche » comme eux.

\section{Chez les Abisi du plateau de Jos au Nigéria}

L'étude des Javanais apparaît plutôt improvisée et inductive : la collecte des données est spontanée, en fonction des observations de tout moment et les thèmes d'analyse se développent à mesure qu'on progresse dans l'accumulation de ce matériel. L'étude des Abisi apparaîtra plus expérimentale, car une problématique générale avait précédé le terrain. Cependant, nous verrons qu'en réalité la recherche anthropologique exige l'usage des deux approches: l'une inductive et l'autre déductive.

Ma motivation à faire un doctorat et celle d'aller en Afrique me sont venues au contact de J.-C. Muller, professeur au Département d'anthropologie de l'Université de Montréal. Comment cet intérêt s'est-il concrétisé ? Assis derrière un temple hindou à la pointe extrême du Sud de l'Inde et face à l'Océan Indien, je venais de terminer une expérience pilote pour voir si la recherche en Inde m'intéressait. J'avais déjà en tête deux projets possibles : soit une étude de l'ancien comptoir français de Pondichéry, soit une étude des cornacs, soigneurs d'éléphants. C'était juste avant d'abandonner l'idée de me rendre chez les chasseurs d'hippopotames du Sud de l'Éthiopie.

En y réfléchissant, je me rendis compte de l'ampleur des acquis préalables pour me spécialiser dans les études indiennes, car religion et société étant intimement liées, il me semblait que plusieurs années d'études théoriques de l'hindouisme étaient nécessaires pour émerger de l'ignorance. De plus, pendant trois années, j'avais « évolué » au sein de la recherche orientaliste et je ressentais un besoin de renouvellement. C'est au Nigéria que j'ai pu le trouver, d'autant plus aisément 
que je préférais d'une part, le terrain au travail en bibliothèque et d'autre part, l'ethnographie d'une petite population, pouvant être faite par une seule personne, à l'étude d'un groupe intégré à une société archicomplexe et explosive.

Le plateau de Jos est une élévation montagneuse au centre du Nigéria en Afrique de l'Ouest. Il s'agit du seul endroit au monde où, sur un même territoire donné, on trouve la plus grande diversité de peuples parlant le plus grand nombre de langues différentes. De plus, dans un milieu physique identifié, des dizaines de populations se sont élaborées en sociétés distinctes sur plusieurs plans : certaines s'étant constituées en royaume ou en fédération de royaumes, en chefferies et d'autres étant segmentaires, sans centre unique de pouvoir. C'est donc un vaste laboratoire où des humains ont expérimenté diverses formes d'organisation sociale et dont il n'y avait que très peu de documents ethnographiques de valeur scientifique.

Il est facile d'imaginer la problématique que soulève une telle région : comment expliquer cette variation sociale dans un milieu comparable ou, en d'autres termes, quelles sont les relations entre l'insertion de ces peuples au sein de leur environnement (les rapports techno-environnementaux) et leurs institutions économiques et politiques (leur structure sociale ou mode de production)?

Au début des années 70 , ce genre de problème était très à la mode, des gens comme M. Godelier, C. Meillassoux et d'autres, anthropologues américains, discutaient des relations écologico-économiques. Les deux années nécessaires à la préparation de ce terrain me plaçaient au centre de ces débats qui comprenaient d'autres questions soulevées par les anthropologues britanniques, dont l'influence au Nigéria est très importante, et par les ethnologues français de l'école structuraliste.

C'était une époque de grands débats, mais les études doctorales, au Québec comme ailleurs, probablement, manquaient d'encadrement universitaire. Il y avait peu de cours et rares étaient ceux pertinents à la recherche de l'étudiant. Heureusement pour moi, il y avait à cette époque deux professeurs dans mon département, Muller et Berthoud, qui étudiaient ces questions au Centre Nigéria et c'est beaucoup plus par des échanges avec eux que je développai une formation appropriée 
à ma recherche. Cette préparation consistait à lire et à discuter des problèmes théoriques généraux, des méthodologies et surtout, à assimiler l'information ethnographique sur l'Afrique, le pays et la région où je voulais travailler. Comme la problématique exigeait une préparation à caractère écologico-économique, j'étudiai, par exemple, comment analyser la production agricole. Il fallait se familiariser le plus possible avec les différentes espèces de plantes cultivées, les sols, le climat, les moyens de travail, la tenure foncière, les relations de travail, les rapports de production : qui dirige ? qui contrôle les produits ? comment sont-ils distribués, consommés ? dans quels cadres ? Une longue liste de questions de cette nature fut préparée. Il ne me restait qu'à choisir une population.

En discutant avec J.-C. Muller, nous avons d'abord pensé que toutes ces populations inconnues des ethnologues étaient aussi intéressantes les unes que les autres et que finalement, je pouvais choisir au hasard un peu comme si en mettant la carte de distribution ethnique au mur, je pouvais lancer une fléchette afin de déterminer mon choix. Cependant, Muller me dit qu'il savait qu'au moins quelques personnes chez des voisins des Rukuba (là où lui-même travaillait), les Piti ou Abisi, voulaient un ethnographe. En effet, un jour que Muller participait à une cérémonie de chasse interethnique, des Abisi apprirent qu'il s'agissait là de son travail d'ethnologue. Intéressés, ils lui demandèrent de venir les voir, car ils étaient certainement les plus captivants de la région. Muller leur dit qu'il essaierait plutôt de leur trouver quelqu'un dont ils pourraient eux aussi disposer comme les Rukuba le faisaient avec lui. Cette information me convainquit qu'il ne fallait pas décevoir ces gens, d'autant plus que les brèves descriptions de l'anthropologue colonial C. K. Meek ${ }^{1}$ étaient stimulantes.

J'ai donc fait une demande de permis de séjour au Nigéria pour deux années. Neuf mois plus tard, il n'y avait toujours rien : le consulat du Nigéria ne pouvait encore délivrer de visas. Pour entrer dans ce pays au-delà de trois mois, il faut soit être invité par le gouvernement,

1 MEEK, C.K. (1925), The Northern Tribes of Nigeria, London, Oxford University Press, 2 volumes.

MEEK, C.K. (1931), Tribal Studies in Northern Nigeria, London, Trench, Trubner and Kegan, 2 volumes. 
soit être employé par une entreprise installée sur place. Je ne répondais pas à ces exigences. Malgré divers contacts au gouvernement, les visas étaient retardés. Chaque téléphone à Ottawa était esquivé par un laconique : « Not yet ». En septembre 1972, j'avais déjà perçu 3 500\$ et le prix de mon billet d'avion du Conseil des Arts du Canada. Le voyage était planifié en compagnie de mon amie qui s'intéressait à la linguistique. Pressé de toutes parts, je demandai au consulat du Nigéria de me faire délivrer le visa à Paris croyant que c'était une question de quelques semaines seulement. Cependant, il a fallu attendre quatre mois et, grâce à l'intervention de l'ambassade du Canada en France (dont certains membres avaient des «relations » africaines), le permis de séjour fut finalement délivré à la fin de décembre.

Heureux hasard, en me promenant sur le boulevard Saint-Germain au début septembre, j'arrivai face à face avec un Antillais ayant étudié en même temps que moi en anthropologie à Montréal. Dans le grand appartement qu'il partageait avec son frère, il avait l'habitude de recueillir des connaissances du Québec de passage à Paris. Cette hospitalité dura quatre mois, et c'est ce qui me permit d'épargner la plus grande part de ma bourse pour l'Afrique.

À en juger par les normes en anthropologie, mon installation sur le terrain fut très rapide. En deux semaines, j'avais obtenu les autorisations locales et régionales de recherches, établi des rapports avec le musée régional qui me prêtait une petite hutte lors de mes séjours à la ville de Jos et me permit de louer un "Landrover » pour effectuer des allers et retours à toutes les trois ou quatre semaines entre la Ale et la brousse.

Le premier contact avec le village de Warsa Piti, où j'allais résider, s'est effectué par l'intermédiaire de l'ancien cuisinier de mon directeur de thèse. Nous sommes parfis du marché de Jos à la fin d'un chaud après-midi dans l'habituel camion surchargé de produits, de bêtes et de personnes, et nous sommes arrivés au village de Warsa cinq heures plus tard, après $50 \mathrm{~km}$ de piste et au beau milieu d'une fête. Aussitôt descendus avec nos bagages, nous fûmes entourés d'une foule curieuse, très excitée et dont plusieurs personnes étaient en état d'ébriété avancée. À la lueur des torches, parmi les cris et le son des tambours, ce premier contact fut le moment le plus " énervant» de 
mes voyages, partagé entre une certaine angoisse et l'excitation du moment. D'emblée, nous fûmes pris à partie par plusieurs personnes. Que venez-vous faire ici ? Comment se fait-il que vous arriviez du Sud, ici c'est un État du Nord et notre capitale est au Nord ! Problème en apparence mineur, mais exacerbé par les rivalités régionales, auquel notre guide eut tôt fait de couper court en demandant cyniquement : « Do you want to divide Nigeria ? - «Voulez-vous diviser le Nigéria ? » La guerre du Biafra venait de se terminer dans un bain de sang...

Finalement, un jeune homme en "service civil » - les diplômés remboursent leurs dettes d'études en travaillant dans les régions moins développées du pays - comprit les quelques explications que je donnais. Nous allâmes chez le chef administratif qui reporta la discussion au lendemain et nous prêta une case et un lit, et nous fit servir du riz. Le jour suivant, le calme nous permit d'expliquer l'intérêt porté aux Abisi. Je voulais étudier l'histoire, la langue et les coutumes des Abisi et pour cela il fallait que je réside sur place une année ou deux. Le chef n'avait pas d'objection, mais j'appris plus tard que tout le monde croyait qu'un « européen » ne pouvait survivre plus de deux semaines aux conditions de vie de l'endroit.

Après être retourné à la ville chercher le reste de l'équipement, je m'étais installé dans une "maison pour les invités » (rest-house), la seule maison au toit de tôle, située à l'écart. Comme il n'était pas possible d'occuper cette maison pour longtemps et que les maisons abisi sont surpeuplées, le chef administratif me loua un terrain où je fis construire ma propre maison, pour $500 \$$ et dont la majeure partie de la somme servit aux pourboires. Cette maison comprenait une case rectangulaire avec deux chambres, l'une pour dormir et l'autre pour travailler, deux cases oblongues, soit une cuisine et une chambre d'amis, une petite case rectangulaire, constituée d'une fosse septique et d'un lavoir-douche. Elle était construite en terre, recouverte d'un toit de paille et dont la devanture était blanchie à la chaux ; elle était également entourée d'une palissade dont l'entrée était recouverte d'un toit de paille sous lequel on s'asseoit pour discuter à l'abri du soleil. C'était une jolie et confortable installation construite selon les normes. 
Il est normal au Nigéria de parrainer des personnes, de les aider à trouver des emplois moyennant un échange. Le premier guide me demanda, entre autres, d'engager un jeune homme, Yakubu, comme cuisinier.

Je n'avais pas prévu cette dépense et je résistai à sa requête. Ce fut peine perdue, car les pressions furent si fortes que finalement, j'acceptai après avoir compris que je n'avais pas le choix et que la coutume était ainsi. Il importait peu que j'aie ou non l'argent et qu'un tel service soit justifié ... Je n'ai jamais si mal mangé de ma vie ! Ce Yakubu n'avait aucune compétence en cuisine, encore moins en hygiène. Mais le comble fut qu'il profita de son nouveau statut de travailleur pour séduire la fille du voisin. Ce fut le bouquet !

Je discutai avec lui et, par d'autres amis, j'appris qu'il ne faisait que profiter de la situation afin de toucher l'argent nécessaire pour se payer un brevet de chauffeur de taxi. Je lui offris une aide financière à la condition qu'il parte immédiatement pour la ville. Il accepta et nous pûmes ainsi respirer à l'aise et jouir d'une bonne nourriture.

\section{NOURRITURES TERRESTRES}

$\underline{\text { Retour à la table des matières }}$

Toutes les expériences de terrain sont exotiques. L'Amazonie, l'Afrique, Charny ou St-Charles de Bellechasse au Québec, l'ethnologue est toujours quelque peu déphasé par rapport à son objet d'étude. Cependant, la vie quotidienne d'ici ou d'ailleurs n'a pas de commune mesure : les couleurs, les goûts, les odeurs, les sons, l'univers des sens est bouleversé. En fait, mon expérience pédagogique me rappelle l'intérêt que portent les étudiants à ces questions : Comment vous ont-ils reçus ? La chaleur était-elle incommodante ? Qu'est-ce que vous mangiez ? Avez-vous été malade ? Avez-vous eu des relations sexuelles ?

D'une façon générale, je ne suis pas particulièrement incommodé par la chaleur, mais certaines habitudes tropicales contribuent à allé- 
ger des malaises comme l'insolation et la déshydratation. Une bonne adaptation est réalisable dans la mesure où on peut dénicher des endroits ombragés, de l'eau potable et qu'on ait la possibilité de se reposer si le besoin s'en fait sentir. Cependant, cela n'est pas toujours facile ou possible.

Au Nigéria plus précisément, certaines situations furent éprouvantes. Le territoire abisi couvre environ $100 \mathrm{~km} 2$ que l'on doit parcourir en tout sens, si l'on veut obtenir des entrevues ou participer aux événements. Presque chaque jour, je partais à vélo visiter des gens à travers les champs labourés, j'empruntais de petits sentiers élevés, entre 10 et $15 \mathrm{~km}$, pour atteindre les pentes du " haut » territoire, et ce, en plein soleil à une température supérieure à $45^{\circ} \mathrm{C}$. Inutile de le dire, pour une personne pas très sportive, ce terrain fut un véritable conditionnement physique, mais le prix à payer était assez élevé. Combien de fois en arrivant chez moi, les jambes flageolantes, en sueur, la gorge sèche, étourdi et ayant mal à la tête, je m'affalai pendant plusieurs heures afin de récupérer !

En 1973, au Nord du Nigéria, le principal problème était celui de l'eau potable, car cette région avait été touchée par la grande sécheresse du Sahel qui avait fait non seulement des dégâts incommensurables, mais de nombreuses victimes. En saison sèche, le matin vers $8 \mathrm{~h}$, il ne restait plus que de la vase dans la source. Cette eau devait être bouillie pendant vingt à trente minutes sur un petit réchaud "Coleman », et ensuite être filtrée. Parfois, il fallait recommencer le filtrage deux ou trois fois pour enlever la boue qui obstruait le filtre. Cette opération terminée, on obtenait environ cinq litres d'eau potable, bien chaude, que l'on pouvait toutefois rafraîchir en plaçant des bouteilles étanches dans des vases de terre enfoncés dans le sol et contenant une eau non filtrée, mais refroidie par l'effet de l'évaporation. Utilisée pour boire et cuisiner, cette eau était le bien le plus précieux de la maison.

Également, il faut s'adapter aux activités quotidiennes de la population. En général, en Guyane comme au Nigéria, les gens se levaient très tôt, entre 5 et 6 heures, faisaient la sieste l'après-midi, entre 13 heures et 15 heures, et se couchaient vers 23 heures. La sieste est plutôt une bonne qu'une mauvaise habitude à prendre, puisqu'on risque d'être la seule personne debout au soleil au moment le plus torride de 
la journée et sans avoir de denrées ethnographiques à recueillir. De plus, comme les nuits sont courtes, il s'agit d'un très bon moyen d'accumuler la réserve d'énergie nécessaire à un organisme qui tente de s'adapter aux conditions physiques du milieu !

Celle-ci ne se fait pas sans heurts à cause des micro-organismes tropicaux. Si en Guyane je n'ai pas eu à souffrir de quoi que ce soit, il en fut autrement au Nigéria. Nous eûmes effectivement à faire face à de nombreux et sérieux problèmes de santé. D'une part, dès notre arrivée mon amie contracta une dysenterie amibienne. D'autre part, j'eus une crise de paludisme et une entérite chronique et, pour couronner le tout, une infection de type typhoïde, dont la variante était inconnue, nécessita une hospitalisation de mon amie qui, d'ailleurs, faillit y laisser sa peau. Parfois, l'embêtant ce n'est pas tant la maladie elle-même, mais davantage la fatigue et les limites de l'organisme.

Dans ces contrées, il n'y a pas que les petites bêtes, mais il y a aussi les moyennes et les grosses. La prudence et la chance nous permettent de ne pas marcher sur un scorpion ; mygales et serpents se rencontrent fréquemment. Les exemples d'événements angoissants ne manquent pas : une mygale indécise tout près de mon épaule, un serpent venimeux dans la boîte servant de réserve alimentaire, ou se lovant autour de la patte de table devant laquelle quelqu'un était assis, ou encore cet autre serpent paré à frapper d'un point stratégique dans le toit de paille servant de portique à la maison. Avec le temps on s'habitue, on en vient à considérer ces bêtes comme des proies et avec la hache ou le javelot, on passe à l'action prenant exemple des jeunes Abisi qui, aussitôt le serpent tué, le font griller sur un petit tas de branchailles et le dégustent...

Sur le plan alimentaire, il faut recommander à tout gastronome de s'orienter surtout vers les civilisations orientales. Habituellement, chez les Javanais de Guyane par exemple, on cuit du riz pour le repas du soir, on le consomme avec du poisson ou du poulet et quelques légumes verts. Le riz qui reste est conservé pour le petit déjeuner, et le midi on mange des plats variés composés d'oeufs, de manioc, de volaille, de poissons ou encore d'excellentes soupes. Souvent aussi, en saison, les repas sont à base de divers gibiers tels que l'iguane (et ses oeufs), le tapir, le « cochon-bois » (espèce de sanglier), la biche, l'agouti, le 
savoureux caïman et divers oiseaux tropicaux aussi beaux à regarder que savoureux à déguster !

Ce quotidien culinaire est remplacé en de multiples occasions, particulièrement lors des fêtes religieuses appelées Slametan, festins gastronomiques que l'on partage avec les esprits et par lesquels on recherche l'harmonie, c'est-à-dire le Slamet. Chaque famille cuisine une variété de plats que l'on apporte chez les hôtes de la fête et selon certains rites, les invités repartent avec différents plats qu'ils consomment ensuite chez eux.

Au Nigéria, l'alimentation diversifiée n'est pas une priorité. Graminées et farines diverses accompagnées de sauces piquantes constituent l'alimentation de base. Pour ma part, je me suis surtout alimenté de conserves, de spaghetti, d'œufs de volailles diverses, que les enfants pouvaient trouver, et d'ignames. La spécialité régionale est une bonne bière maison servie dans une calebasse et recouverte d'une couche de piments rouges forts. Selon la coutume, cette bière se boit avec une autre personne, joue contre joue et simultanément d'un seul trait. Après quelques calebasses d'un litre chacune, non seulement on est un peu soûl, mais on envahit les lieux isolés...

La vie affective des ethnologues sur le terrain est un sujet tabou. On peut toutefois dire qu'il est fréquent qu'ils ou qu'elles aient des fiancé-e-s et cela pose souvent d'énormes problèmes.

Il faut se rendre compte que dans une petite communauté il n'y a aucune intimité, tout se sait et ce n'est pas fortuit si les ethnologues reconnaissent le commérage, la médisance et la calomnie comme d'excellents moyens de contrôle social. Toute relation entre les sexes est régie, selon les cultures, par un code particulier dont on ne peut difficilement déroger même si l'on est étranger. Danser deux fois avec le ou la même partenaire dans une soirée équivaut à des fiançailles ; accepter un cadeau peut être un engagement au mariage.

Comme nous le verrons plus loin, l'ethnographie des Javanais était sous le signe de l'ascétisme et les conditions de ce terrain ne permettaient pas de déroger à cette règle. Cependant, je développai une amitié intime avec une jeune fille du village et il n'en fallut pas plus pour 
que les parents fassent des plans et exercent des pressions pour qu'éventuellement nous partions ensemble pour le Canada. Comme ni l'un ni l'autre n'étions intéressés à dépasser cette relation d'amitié, les parents déçus eurent recours à d'autres moyens dont l'utilisation de certaines amulettes (fétiche); la dernière ayant été découverte longtemps après mon retour du terrain. Cachée dans le repli d'un chapeau, j'ai trouvé une mèche de cheveux enveloppée dans un papier couvert d'inscriptions magiques !

La situation au Nigéria était différente, car je n'étais pas seul. Cependant, il n'aurait pas été difficile de trouver une compagne, car les femmes abisi sont polyandres... ma monogamie fut d'ailleurs l'objet de railleries autant des hommes que des femmes.

Toutefois, la dimension affective s'est révélée sur un tout autre plan que sexuel.

Comme les parents ne tolèrent pas les enfants bruyants à la maison, ceux-ci profitèrent de mon statut de marginal pour envahir ma cour intérieure qui devint leur lieu de rencontre. J'appris à vivre avec les enfants et même avec les tout-petits qui venaient pour avoir des friandises et pour s'asseoir à mes pieds pendant que j'écrivais. D'ailleurs, ces relations furent, pendant un certain temps, centrées sur les pieds. Pour expliquer cet attrait, il suffit d'observer le pied d'un adulte africain qui a marché toute sa vie sans chaussure et dont la corne est si épaisse qu'elle fait un important rebord qui lui sert à se protéger lorsque, pieds nus, il éteint les braises. Cette différence n'échappait pas à mes jeunes invités qui, tour à tour, s'amusaient à me caresser les pieds dont la beauté, disaient-ils, était exceptionnelle. De telles découvertes physiques réciproques étaient courantes, car la plupart d'entre eux n'avaient eu aucun contact avec des descendants \&Européens. Ces caresses et ces échanges affectueux contrastent avec ce qu'on retrouve dans d'autres cultures et c'est peut-être ce qui détermina mon désir d'avoir des enfants à mon retour.

Cependant, comme dans toutes choses, l'abus est néfaste ; ma maison est rapidement devenue un lieu où il était impossible de s'entendre parler au milieu des cris des jeunes excités et des autres qui, parfois tard le soir, sous ma fenêtre de chambre, pratiquaient le tambour. 
Après avoir tenté en vain de convaincre les enfants de jouer ailleurs et après avoir essayé de leur faire peur, je me couvris de ridicule. Alors, je demandai l'aide du chef qui m'assura une certaine tranquillité. Les enfants étaient toujours les bienvenus, mais je restais maître de fermer boutique quand je le désirais. Mais, plus le temps passait, moins je devenais un objet de curiosité et graduellement, rares furent les personnes qui vinrent m'observer pendant que je dormais ou allais à la toilette ...

\section{L'ETHNOGRAPHIE}

\section{Ma relation avec le gourou}

\section{$\underline{\text { Retour à la table des matières }}$}

Le contexte général de l'ethnographie chez les Javanais de Guyane était relativement simple, car cette petite communauté bien structurée se laissait aisément décrire sur les divers plans fondamentaux tels que la répartition spatiale, la famille, le travail et la production, les relations interethniques, l'ethnohistoire, etc. La principale difficulté fut la langue. Mais, comme certaines familles résidaient depuis déjà douze ans en Guyane, plusieurs jeunes enfants parlaient le français et les autres, adolescents et quelques rares adultes, parlaient un peu le créole guyanais. Quand c'était nécessaire, je me faisais accompagner par un interprète que les parents enjoignaient fortement de m'aider, car l'obéissance aux parents est absolue. Malgré cela, la communication n'était pas toujours facile. En effet, dans cette communauté de quelques centaines de personnes, on retrouvait les langues suivantes: le français, le créole, le hollandais, le takitaki, le javanais bas et le javanais haut, l'indonésien et même quelques autres langues indonésiennes différentes du javanais. Parfois, selon les cas, les conversations se déroulaient simultanément en trois ou quatre langues.

Cette ethnographie générale me permit d'identifier rapidement l'un des thèmes clés de la culture, soit les rapports idéologiques ayant présidé au regroupement de ces familles et les mécanismes sociopolitiques maintenant le dynamisme de son organisation. En d'autres 
termes le leadership politique et son assise religieuse se démarquaient comme facteurs principaux dans l'explication de l'existence de cette communauté. Le coeur des phénomènes religieux et idéologiques est constitué de discours, c'est-à-dire d'un système de pensée, de catégories mentales, de croyances dont l'ethnologue peut obtenir diverses versions par des entrevues et en observant, entre autres, les pratiques rituelles. Afin de donner une idée des divers éléments des différentes idéologies existantes dans le village, notons qu'on y retrouvait certaines influences hindouistes, bouddhistes et musulmanes. Ces trois traditions s'intégraient et rivalisaient avec les croyances animistes, avec les diverses formes de christianisme et même avec la critique d'origine marxiste et maoïste. L'axe principal de la relation idéologico-politique dans le village était l'Agama Djawa, synthèse philosophique hindoubouddhiste appelée la « religion javanaise ». Cette religion comprend d'une part, des rites intégrés aux divers moments de la vie et d'autre part, des explications concernant l'origine de l'humanité, ce qu'elle est, son destin. Ces connaissances et leurs dimensions pratiques font partie de la vie quotidienne : des consultations chez le devin sont exigées pour entreprendre un voyage ou tenir une cérémonie.

L'individu est constamment dominé par la présence d'êtres invisibles, mais il peut les contrôler moyennant un certain savoir. Ce dernier se transmet tout d'abord, à l'intérieur du cadre familial et villageois, mais toute personne peut, si elle le désire, dépasser cette information diffuse et recevoir une formation spéciale auprès d'un gourou. Dans ce cas, la connaissance se forge toute la vie durant pour atteindre la sagesse chez la personne âgée. Le corps de l'individu doit devenir un réservoir de force et d'équilibre utilisé efficacement pour le bien de tous et pour influencer les puissances universelles. Cette force physique et morale s'obtient par le jeûne, la méditation et la bonté, mais aussi par la connaissance du monde invisible de façon à utiliser les forces magiques pour combattre le mal et faire le bien. L'ascétisme renforce le corps et lui permet de résister aux forces du savoir qui, une fois apprivoisées, peuvent être utilisées par la personne âgée. Chaque pratiquant fortifie son âme à un état de perfection comparable à celle de Dieu. Cette formation ne peut s'acquérir sans l'encadrement d'un maître a penser, car la moindre erreur de jugement peut mettre en péril la vie même des gens que l'on veut aider. 
L'ethnographie de cette dimension de la culture javanaise exige donc de participer dans une relation de maître-étudiant. En Orient, la relation avec le gourou est généralement caractérisée par la domination du maître qui exige de son disciple une obéissance absolue en retour d'une pension et de conseils. En Guyane, ce sont surtout des personnes dépressives ou malades qui viennent consulter le gourou, mais un certain nombre d'entre elles prolongent cette relation après la guérison et leur retour à domicile.

Comment l'ethnologue peut-il entrer dans cette relation ? Il n'y a ni procédure ni recette véhiculées par l'université. Tout se joue sur la bonne foi et la sympathie des participants. Or il y a là un écart : le gourou présente une approche contradictoire avec celle du chercheur. Sa vision du monde est au-delà de la raison humaine alors que pour le chercheur, la raison peut rendre compte de tout système symbolique. Peut-on entrer de bonne foi dans le mystère et dans l'«irrationalité » ? Contrairement à l'ethnologue qui pense que le discours de l'informateur sera de toute façon neutralisé par ses schèmes d'analyse et qui conserve une distance émotive et philosophique maximale entre ses informateurs et lui-même, j'ai trouvé de multiples points de ressemblance entre le discours du gourou et le mien, " personnel » et « anthropologique ». Il ne s'agit pas de penser que j'ai « cru » que Vishnou était une entité existante et agissante sur mon comportement, mais plutôt qu'il est possible de décoder une rationalité derrière les symboles et de la confronter à ses propres opinions. Ainsi, la recherche de l'harmonie dans les relations interpersonnelles, le développement d'une discipline personnelle, le respect et l'amour de la nature, la lutte contre l'injustice étaient tous des thèmes communs.

La relation qui s'établit avec un gourou est un dialogue pédagogique où l'étudiant doit faire preuve de sa compréhension et de son obéissance, c'est-à-dire mettre en pratique les prescriptions proposées. L'une de ces prescriptions était de me laisser pousser l'ongle du pouce droit pour indiquer mon appartenance religieuse. Une autre prescription était de me comporter de façon ascétique (jeûne, calme, méditation), les journées correspondant à mon ascendance astrologique. Celle-ci est calculée par un système javanais appelé Pétang. La première fois que ce calcul fut fait, on m'a fait pénétrer dans une petite chambre, la "Chapelle de Bouddha », où s'étalait sur une table une 
panoplie d'objets symboliques disparates en apparence : le culte des ancêtres était représenté par des poignards ancestraux (kriss), par de multiples symboles politiques, indonésiens et français, ou par des symboles économiques (pièces de monnaie, gerbes de paddy, semences) et par beaucoup d'autres choses. Je devais répéter trois fois à voix basse : « je demande à mon père et à ma mère, à leurs pères et mères et à tous mes ancêtres de m'aider ». Pendant ce temps, une petite ampoule contrôlée de l'extérieur faisait un effet stroboscopique. Après avoir choisi au hasard une pièce d'un jeu de domino éparpillé sur la table, je la sortis et le gourou calcula mes coordonnées à l'aide de ma date de naissance et des nombres inscrits sur le domino. J'avoue que c'est avec une certaine difficulté que je réussis à conserver le sérieux qu'exigeait la situation. Cela allait toutefois être déterminant dans ma relation avec le gourou : tous deux nous correspondions à l'influence de Vishnou, la divinité généreuse du panthéon. Notre situation se situait donc sur sa meilleure trajectoire, puisqu'elle suggérait que le gourou puisse se reproduire intellectuellement ou non !

Ainsi, trois soirs par semaine, les journées de Vishnou, je me retrouvais en tête-à-tête avec le gourou. Ces journées étaient les points forts du terrain, car pendant plusieurs heures d'entretien, je pouvais avoir un informateur disponible qui autrement, comme dans toute société paysanne, était occupé toute la journée. Ces entretiens se déroulaient en trois phases : un discours du gourou, des questions et des réponses sur le discours et sur les observations quotidiennes. Cette relation implique l'assimilation rapide de l'information, car il est inutile que le maître poursuive si chaque étape n'est pas bien comprise. En général, la soirée se terminait au son d'une musique indonésienne enregistrée et parfois même, par une chorégraphie traditionnelle que le chef exécutait devant la famille.

\section{L'ethnographie des Abisi}

Ayant déjà poursuivi une correspondance avec un certain Raga, jeune homme intéressé à servir de guide et à travailler avec moi, je fis des arrangements et négociai de façon à établir un programme commun de travail et un salaire mensuel. Ces arrangements menèrent fréquemment à des discussions, en particulier lorsque des jeunes tentèrent de convaincre mon ami que les Blancs étaient tous très riches. 
Une personne me montra d'ailleurs la photo d'un édifice à bureaux de Londres, persuadée qu'au Canada je possédais une maison semblable avec plus de 300 chambres! Ces discussions furent plutôt pénibles. J'avais établi un budget de fonctionnement: résidence, transport, nourriture, interprète, argent de poche, etc. Tout ce qui n'était pas essentiel était investi pour le terrain et la majeure partie de cette somme était allouée à l'interprète que je payais le même salaire que celui d'un instituteur, puisque ses tâches étaient définies dans les mêmes termes.

Ce n'est qu'après plusieurs mois que je réussis à convaincre Raga que je n'avais qu'une somme fixe allouée pour toute la durée de mon séjour. En fait, c'est en résistant au chantage que je réussis à gagner mon point. Depuis plusieurs mois, j'attendais afin de participer aux cérémonies de mariage, et quand l'agitation et le tambour annoncèrent qu'une cérémonie aurait lieu, Raga refusa de m'accompagner. Compte tenu que je considérais pouvoir me débrouiller seul, je suivis un convoi qui m'amenait au lieu du rite et à la fin de la soirée, je suivis un groupe d'enfants qui revenaient à mon village. Il faut se rendre compte que, quoique relativement petit, le territoire abisi est sillonné de toutes parts par de multiples sentiers qui mènent dans une jungle de grandes herbes de trois mètres de hauteur. Le soir et surtout la nuit, en période nuageuse et de pluie, il est facile de s'y perdre.

Le jour suivant, je vis mon copain arriver chez moi à $7 \mathrm{~h} 30$, prendre son " Canadian Tea », un café apparenté au moka, c'est-à-dire additionné de chocolat et de lait en poudre. Mine de rien, il me dit que l'on devrait aller le soir même voir un grand regroupement matrimonial ! Il avait compris que je pouvais me passer de lui pour me déplacer et pour parler un tant soit peu avec les gens. Je n'ai plus jamais entendu parler des sommes faramineuses dont je disposais pour faire ce travail. Cependant, la relation d'intérêt prit une autre tangente : prêter de l'argent pour l'achat d'un vélo, d'un lit et surtout, pour un mariage.

En général, mes relations avec les gens portaient rarement sur des questions d'argent. Parmi plus d'une centaine d'informateurs et d'informatrices avec lesquels j'ai travaillé, la question d'argent fut complètement absente. Parfois, le simple fait de parler un peu la langue m'introduisait dans un réseau de sympathie incroyable. D'autres fois, une 
simple connaissance arrivait chez moi, avec une poule attachée au vélo et me l'offrait en cadeau. Connaissant la règle de la réciprocité, je ne manquais jamais de donner un contre-don en nature à tous ces gens et chaque fois que je me rendais dans un hameau parler à quelqu'un, j'apportais du tabac, des noix de kola et même des bouteilles de jus concentré. Je prêtais aussi un peu d'argent à des gens dans le besoin.

Le rôle de Raga fut considérable dans ma recherche, surtout sur le plan de l'apprentissage de la langue. Pour apprendre cette langue de tradition purement orale, il me fallait constituer quotidiennement un glossaire en phonétique internationale, en recueillant tous les termes techniques liés à l'ethnographie, de même qu'un vocabulaire plus général.

L'abisi est une langue à trois tons. Cela signifie que le même mot prononcé avec un ton haut ou bas sur la première syllabe par exemple, donne un sens différent au mot. Pour l'oreille sans éducation musicale, il est extrêmement difficile sinon impossible de reconnaître le sens d'un mot. Seul le contexte de la phrase peut aider un peu. Même après une année, les gens trouvaient que je parlais comme un enfant -chuinchuin-chuin - disait-on, car je n'arrivais pas encore à prononcer correctement.

Pour les discussions sérieuses, l'aide de Raga était essentielle. Il faut aussi ajouter d'une part, qu'étant un des rares francophones à étudier dans un pays à tradition coloniale britannique, je dus réapprendre l'anglais car le « Nigerian English » est truffé d'expressions locales. D'autre part, le Nord du Nigéria est sous l'influence de la lingua franca qu'est le hausa, et la langue abisi est tributaire de ce bilinguisme.

Malgré les questions monétaires, les relations avec Raga avaient pris une dimension fraternelle et de plus, il considérait son travail d'apprentissage de la culture abisi comme un véritable défi. Somme toute, ce n'est pas parce qu'on naît dans une société, qu'on connaît et interprète de façon satisfaisante les rapports sociaux qui s'y rattachent. Or la plupart de mes découvertes étaient discutées avec lui. Cela allait de l'inventaire spécialisé du système matrimonial dans lequel luimême et ses amis découvraient de multiples aspects pratiques, par exemple, l'inventaire de l'effectif des filles «mariables », à la ré- 
flexion poussée sur les effets de l'intervention de l'État ou des rapports aînés-cadets. De plus, il apprit rapidement les éléments d'ethnologie que je lui enseignais de façon à pouvoir utiliser des catégories communes.

La première règle que nous enseigne l'africanisme britannique est de découvrir le plus rapidement possible la structure sociale : Quels sont les groupes constituants de la société ? Comment sont-ils reliés les uns aux autres ? Pour ce faire, je partis visiter une première maison qui fut, en l'occurrence, celle de l'oncle maternel de Raga. L'oncle maternel est considéré comme la personne avec laquelle on se sent le plus à l'aise, dont on peut exiger tout ce qu'on veut et qui, par surcroît, pardonne tout. C'était en quelque sorte un test pour vérifier comment je me comporterais en visite de travail chez des gens sans que Raga ne se sente trop intimidé.

La formulation de ce problème fut assez difficile, car les termes spécifiques correspondant à nos notions ethnographiques de groupes, de clans, de familles, etc., recouvrent des réalités fort différentes. « How are you divided ? » Je découvris que les Abisi distinguaient les groupes par des noms de lieux, par exemple « les gens de la grosse pierre », selon les noms de personnes, " les gens de Mali », selon des noms ethniques et aussi selon l'appartenance à des sections rituelles. Mon premier travail fut donc de démêler tout cela, tout en apprenant à m'orienter physiquement dans le labyrinthe des sentiers et à cartographier mentalement les groupes résidentiels. Pendant quelques semaines, je parcourus le territoire et je m'introduisis aux gens des divers hameaux en leur expliquant mes buts tout en élaborant l'analyse démographique. Dans certains hameaux, l'arrivée d'un Blanc faisait fuir les enfants qui, apaisés, s'approchaient ensuite avec un air de curiosité. Cette peur n'était pas injustifiée, car ils avaient entendu parler des " enus », esprits de blancs colonialistes venus au début du siècle et que certains parents utilisent pour contrôler les plus turbulents : «Si tu n'es pas tranquille, le Blanc va t'emmener et te manger (Croc !). »

Cette anecdote indique que le statut de l'ethnologue dans une communauté est controversé. Dans plusieurs situations, on va chercher à l'éprouver pour tester ses limites physiques et intellectuelles. Une fois en particulier, on a voulu me faire monter le grand cheval de 
l'Émir, on croyait s'amuser à mes dépens. Jamais je n'ai monté avec autant de plaisir une bête aussi obéissante ... les mors de chevaux hausa ont des petites pointes d'acier qui piquent sous la langue. Pour les Abisi, ce test fut significatif, car eux-mêmes sont de bons cavaliers.

Le débat de fond à mon sujet a porté sur ma nature « humaine » ou « inhumaine ». Pendant un certain temps, j'entendais prononcer autour de moi le terme «Okap ». J'appris qu'on utilisait le même terme pour parler des babouins et des divers singes ! Évidemment, je me défendis d'appartenir à cette espèce zoologique, mais il faut admettre que certains arguments étaient bien fondés ! En effet, qui d'autre qu'un singe a les jambes poilues, qui d'autre qu'un singe mange le maïs en croquant les grains directement sur l'épi, alors que toute personne éduquée enlève les grains de l'épi avec les doigts et les porte ensuite à sa bouche! Pour comble, des bribes de discussions philosophiques avec des créationistes musulmans se répandirent chez quelques-uns : je ne croyais pas à la création, mais à l'évolution de l'humain en tant que primate. Je me croyais donc un singe ! Le fait d'écrire m'aida quelque peu à réhabiliter mon image.

En fait, mon statut s'est défini graduellement sur la même base que les autres. D'abord, j'appartenais au groupe de mon village et à la section de Raga, puisque je l'accompagnais souvent. Je devais donc respecter les droits et les devoirs de ce groupe. J'appartenais ensuite à un groupe d'âge. Cette catégorie est fondamentale chez les Abisi. Au début, je portais la barbe et j'étais invité à m'asseoir parmi les plus âgés. Quand j'appris que la barbe était réservée aux plus vieux seulement, je me rasai, ce qui occasionna l'interdiction de m'asseoir avec les plus vieux et me fit reléguer dans mon groupe d'âge. J'étais finalement considéré comme un dilettante assez riche pour séjourner chez eux sans faire d'agriculture et qui s'avisait d'écrire l'histoire des Abisi. Au contraire des Javanais, je ne fus pas ici intégré en tant que «fils de... ", mais en tant qu'immigrant au même titre que plusieurs ancêtres des Abisi qui étaient aussi des étrangers.

La principale difficulté de l'analyse des données fut d'établir l'inventaire socio-démographique et l'esquisse du système de descendance, car le moment était mal choisi. Pendant toute la saison sèche je n'entendais parler que de chasse et de guerre. On me dit: "Mais 
pourquoi nous parles-tu de ces choses, segmentation, fission, descendance, alors qu'ici on ne parle que de chasse ? » J'appris alors qu'il fallait vivre le moment présent et que cela ne me donnait rien de tenter d'aller à l'encontre du calendrier annuel des activités, d'autant plus que souvent on disait « oublier » les événements qui n'étaient pas en cours et on se demandait pourquoi se faire expliquer quelque chose qu'on a pas vu?

Ici, l'empirisme domine les plans de recherche les mieux élaborés et je dus attendre pour réaliser les enquêtes socio-économiques. N'eût été le fait de ma présence tout au long d'un cycle annuel complet, il n'aurait pas été possible de réaliser mon projet de monographie. Si l'on peut décrire le procès de la chasse, par exemple, il est impossible d'en comprendre la portée sans connaître la division du travail agricole que l'on apprend quelques mois plus tard, puis le régime matrimonial qui s'observe huit mois plus tard, et finalement c'est au bout d'une année que les réponses aux questions du début trouvent leurs fondements.

La méthode de recherche que j'ai utilisée tout au long du terrain peut être répartie en six phases. La première était obligatoirement l'observation des activités en cours. Cela non pas pour des raisons abstraites ou par empirisme inconditionnel, mais bien, comme nous venons de le voir, parce qu'il était inutile de se situer hors de l'action sociale en cours. Ainsi, par exemple, le premier mariage auquel j'assistai m'indiquait que le temps de l'ethnographie de l'alliance était commencé. Tous les mariages ont lieu en août seulement et si, par malheur, l'ethnographe n'est pas à son poste, il devra attendre une année pour en discuter. Cette phase d'observation est aussi une phase de participation dans la mesure où l'on joue un certain rôle dans l'activité. Donner un coup de main dans le transport des choses, aider les rabatteurs à la chasse peuvent toujours se faire mais, mises à part certaines épreuves auxquelles il faut se prêter, il y a trop à faire pour vraiment s'impliquer dans toutes les activités surtout si l'on songe au faible niveau de compétence de l'ethnologue. Sauf peut-être en ce qui concerne la consommation de bière locale en groupe.

Les observations sont ensuite utilisées pour des schémas d'entrevues. Il s'agit de poser toutes les questions possibles aux participants des activités et de reprendre une à une les observations et de se les 
faire expliquer : Qui étaient les gens présents ? Pourquoi tel ou tel acte a-t-il été posé ? À partir de ces données, il faut développer une problématique particulière aux événements, chercher à en imaginer les ramifications, à vérifier les observations. À ce niveau, la connaissance de l'ethnographie générale devient très utile car, par la comparaison, on peut formuler des problèmes inédits. Cela se révéla d'autant plus dynamique que les Abisi eux-mêmes utilisent parfois cette procédure comparative pour expliquer leurs propres coutumes. Ils diront par exemple : " Ici on est pas comme chez les Rukuba, nos filles ne vont pas avec les garçons avant de se marier. » Il faut ensuite élaborer un instrument de recherche spécialisé et faire la collecte systématique des données. Finalement, l'analyse de ces résultats permet de poursuivre la recherche en rattachant chaque secteur étudié aux autres.

Par exemple, après avoir observé les groupes de travail agricole, après avoir interrogé les participants sur la nature et le fonctionnement de l'institution, j'en arrivai à élaborer une problématique sur les rapports travail-mariage. Ensuite, je développai un questionnaire pour faire l'inventaire des prestations de travail pour les mariages auprès d'un échantillon de familles ayant reçu ce type d'aide. Cependant, tout n'est pas si facile, car ces études précédaient la période des mariages dont je ne comprenais pas très bien le fonctionnement. Je dois dire que le "sexo-centrisme " tant de l'ethnologue que des hommes abisi retarda cette découverte. En effet, comme ce ne sont que les hommes qui font les labours, je démarrai cette recherche avec des informateurs masculins. Pendant des semaines, j'essayai de comprendre le système matrimonial. À l'aide d'un échantillonnage de réseaux matrimoniaux polygyniques des hommes, j'essayai de cartographier ces échanges et d'en construire un modèle graphique. J'obtins une sorte de sociogramme liant les divers hameaux, mais qui avait plutôt l'air d'avoir été tracé avec un « spirographe ». Cela ne me permit pas d'en dégager le système.

Il est courant chez les femmes abisi d'avoir un ami personnel avec lequel elles échangent de l'aide et de la sympathie. J'avais une telle amie qui me parla d'un homme avec lequel elle avait des problèmes. C'était, dit-elle, l'un de ses époux qui voulait qu'elle aille vivre chez lui. Les femmes abisi ont trois ou quatre maris chez qui elles ont une chambre et des possessions et où elles peuvent retourner si elles le 
désirent, car il n'y a pas de divorce. C'est d'ailleurs l'un des mécanismes qui empêche la violence envers les femmes, puisque chacune d'elles peut compter sur d'autres époux qui pourraient sévir contre celui d'entre eux qui lui ferait violence. En discutant avec cette amie, j'appris de quel groupe social étaient issus ses différents époux. Je découvris alors la structure matrimoniale: par ses mariages, toute femme peut avoir accès aux cinq sections de cette société, mais cette structure lui interdit d'épouser plus d'un homme originaire de chacun de ces groupes. Ensuite, je pus rapidement analyser ce système et réorienter mes enquêtes auprès des femmes, dont 75 répondantes, qui me permirent d'en faire l'analyse statistique. Le sexo-centrisme habituel m'avait fait perdre un peu de temps et il avait aussi failli me faire rater la découverte de ce nouveau modèle matrimonial.

Au niveau technique, je consignai mes observations dans des blocs-notes ou des cahiers. Les entrevues les plus formelles étaient également enregistrées au magnétophone, puis traduites et transcrites avec l'aide de Raga. Les observations que le contexte ne permettait pas de noter directement l'étaient sur de petits blocs-notes de poche dès que l'occasion se présentait. Parfois, je devais faire semblant d'aller derrière la maison par force majeure et noter rapidement les points que j'avais peur d'oublier. Toutes ces données étaient ensuite analysées, puis transcrites par segments sur des demi-pages en plusieurs copies dont une était rapidement enveloppée de plastique, placée dans une boîte de nylon et expédiée le plus tôt possible à Montréal, par crainte de les perdre par le feu, l'eau ou les insectes. Les autres copies d'observations ou d'entrevues étaient classées sous diverses rubriques, par exemple, économie et parenté, chacune subdivisée par sousthèmes : travail, agriculture, chasse. Au fur et à mesure que la problématique de la recherche se raffinait, ces rubriques étaient réaménagées selon les chapitres éventuels de la monographie. 


\section{CONCLUSION}

$\underline{\text { Retour à la table des matières }}$

D'un point de vue global, le terrain de l'ethnologue est considéré comme la plus belle illustration et comme la plus nette contradiction du principe de Peter selon lequel le personnel des systèmes hiérarchiques se hisse à son niveau d'incompétence. L'arrivée sur le terrain m'a fait penser que j'étais devenu l'être humain le plus incompétent sur terre. Je ne savais plus ni me comporter, ni parler, ni manger, ni boire, ni même faire mes besoins. Plusieurs de ces apprentissages doivent être réappris. Heureusement que le désir de l'anthropologue et les moyens psychologiques et intellectuels à sa disposition lui permettent finalement de développer un minimum de compétence et de paraître de moins en moins comme le clown dont tout le monde rit.

Il faut préciser que plusieurs des personnes rencontrées sur le terrain cherchent aussi à profiter au maximum du passage de l'ethnologue et ce dernier peut être enclin à se justifier en tentant de reprendre un peu de ce qu'il a cédé et ce, non seulement en termes matériels. Si on nous enlève toute vie privée parce qu'exotique, intéressant et drôle à observer, n'est-il pas juste d'entrer dans la réciprocité au même niveau et de prendre aussi un peu des «secrets » des gens ? D'un point de vue personnel, je ne me suis jamais senti ni pensé coupable d'une sorte de viol de l'Autre car, au-delà des justifications idéologiques, je crois qu'il est aussi intéressant pour l'Autre d'observer cet étranger et de communiquer avec lui comme c'est le cas pour l'ethnologue. Chaque personne tire profit de cette expérience interculturelle.

Cependant, il faut voir un peu plus loin que la relation ethnologique et se demander comment justifier ces recherches. Pour ma part, je considère comme une tâche noble et urgente celle des ethnologues d'utiliser les moyens mis à leur disposition par les sociétés riches dans 
lesquelles ils vivent, pour compléter le plus possible les archives de l'humanité. Si la société industrielle contribue vraiment à la destruction des autres modes de vie, il est nécessaire de participer à notre manière à la réflexion concernant l'histoire de cette transformation.

Dans ce texte j'ai esquissé quelques problèmes de la démarche de terrain comme je crois les avoir vécus. Il en ressort qu'à la différence des expériences de laboratoire ou cliniques, l'ethnographie exige que l'expérimentateur s'insère à l'intérieur du dispositif même de l'expérience, un peu à la façon du "voyage fantastique » dans le corps humain. L'exemple de ma relation avec le gourou est peut-être un peu extrême dans la mesure où, à cette époque, j'étais disponible pour une expérience mystique. L'exemple de l'étude des Abisi montre, par ailleurs, que c'est à la manière d'un entrepreneur que la recherche s'est organisée. Cependant, dans les deux cas, les plans de départ furent passablement modifiés, c'est le moins qu'on puisse dire en ce qui concerne les Javanais. Cela m'a appris à envisager le terrain avec optimisme étant donné que la flexibilité permet toujours d'en tirer le meilleur parti.

La recherche sur le terrain n'est pas l'aboutissement du travail de l'anthropologue, mais plutôt ce qui en favorise le développement. C'est en fait tout le domaine de l'analyse et des débats sur les cultures humaines qui constitue le coeur de la pratique anthropologique.

Il faut ajouter que l'analyse des données peut parfois s'échelonner sur plusieurs années et mener à diverses publications scientifiques. Par exemple, le terrain au Nigéria prend son sens par la contribution qu'il apporte dans l'analyse des sociétés mal considérées par la majorité des habitants de ce pays. En un sens, cette information sera utile à la réhabilitation de l'apport de ces petites sociétés à l'histoire de cet État.

Le cas de la Guyane est différent dans la mesure où diverses missions m'amènent à élargir mon champ d'étude à l'ensemble des relations interethniques. Ainsi, lors de mon dernier séjour, l'actualité de la recherche d'information sur l'immigration haïtienne m'a amené à effectuer une étude exploratoire sur cette question. De plus, comme j'ai pu développer des liens avec l'Association des Amérindiens de Guyane, je tente de mettre ceux-ci en contact avec les seuls autres 
Amérindiens francophones des Amériques, ceux du Québec, de façon à répondre à leurs demandes de conseils d'experts concernant leur cause. C'est donc aussi à long terme que le terrain peut avoir des conséquences intéressantes.

\section{Fin du texte}

